\title{
Implications of Bed Rest for Patients with Acute Deep Vein Thrombosis: A Qualitative Study
}

This article was published in the following Dove Press journal:

Patient Preference and Adherence

\author{
Jian-Mei Gong' \\ Jian-Shi Du ${ }^{1,2, *}$ \\ Dong-Mei Han 2,* \\ 'Nursing School of jilin University, \\ Changchun City, Jilin Province, People's \\ Republic of China; ${ }^{2}$ Department of the \\ Lymphatic and Vascular Surgery, China- \\ Japan Union Hospital of Jilin University, \\ Key Laboratory of Lymphatic Surgery Jilin \\ Province, Changchun City, Jilin Province, \\ People's Republic of China
}

*These authors contributed equally to this work
Correspondence: Jian-Shi Du

No. 965 Xinjiang Street, Changchun City, Jilin Province 13002I, People's Republic of China

Tel +86-133|430|430

Email dujs@jlu.edu.cn
Background/Objective: The recommendation of bed rest for deep vein thrombosis (DVT) patients has changed during the last 20 years, and it has become a concern for researchers. The existing researches on potentially harmful treatment of bed rest for DVT patients focus only on physiological outcomes. This qualitative study explored the implications of bed rest from the perspective of patients with acute DVT. Understanding these implications will provide more evidence on whether bed rest should be used as a medical treatment of acute DVT.

Patients and Methods: For data collection, a descriptive qualitative design utilizing semistructured, in-depth, face-to-face interviews with nine patients with acute DVT was conducted. In order to find the themes and subthemes emerging from the interviews for data analysis, the Colaizzi method, which was suggested by phenomenological methodology, was used.

Results: The four major themes found were physical effects, psychological effects, social effects, and post-trauma growth. These themes illustrated the bed rest experiences of patients and it has a negative impact on the quality of life (QOL) amidst acute DVT.

Conclusion: Bed rest for patients with acute DVT is a physically, emotionally, and socially distressing phenomenon that simultaneously affects QOL and induces post-traumatic growth. We believe that bed rest is not beneficial to the physical and mental health of patients with acute DVT. This study adds to the available evidence on the harmful effect of bed rest as a treatment from the perspective of patients with acute DVT. Further quantitative studies should compare the quality of life and psychosocial status of patients with and without bed rest amidst acute DVT.

Keywords: bed rest, deep venous thromboembolism, qualitative, patient experience

\section{Introduction}

Venous thromboembolism (VTE), which includes deep vein thrombosis (DVT) and pulmonary embolism (PE), is a common problem causing high morbidity and mortality all over the world. ${ }^{1}$ It affects one in 1000 patients (ie, 6.5 million people) globally each year. ${ }^{2}$ A study reported that the morbidity of DVT was $10.2 \%$ in a Chinese hospital. ${ }^{3}$ Although no large-scale survey on the epidemiological data of DVT across community populations of China has been conducted, the overall incidence of hospitalizations due to thrombotic events has continuously increased in recent years. ${ }^{3}$

Bell's study found that $46 \%$ to $60 \%$ of PE originated from lower limb DVT. ${ }^{4}$ In most institutions, the fear of dislodging clots by ambulation is more common than the consideration of thrombus propagation and of recurrence; therefore, bed rest is 
recommended at least for the initial stage. ${ }^{5}$ Absolute bed rest requires that all basic physiological activities be done in bed, with only examinations and treatments as the exceptions. For example, a patient put on bed rest cannot go to the bathroom. He/she also becomes entirely dependent on others to supply his/her needs for daily nutrition and social contact. ${ }^{6}$ Historically, bed rest has been considered in the treatment of patients with acute DVT that is once regarded as one of the most powerful prophylactic measures for PE. ${ }^{5}$

However, some studies have also shown that bed rest has molecular and systemic effects, which ultimately affect functional outcomes in both health and unhealthy people. ${ }^{7}$ Upon outlining the effects of bed rest on the body, Browse and Lipnicki concluded that virtually every system is affected in some way. Generally, bed rest can cause the following: impairment of the cardiovascular, hematologic, musculoskeletal, metabolic, thermoregulatory, and immune systems; neuroendocrine systems; constipation; skin breakdown; impaired sleep; negative psychology; ${ }^{8}$ and functional and cognitive impairment. ${ }^{9}$ There is also substantial research comparing its medical consequences with early ambulation. There have been two randomized trials showing that bed rest as a part of the initial treatment of patients with DVT is not able to substantially reduce the incidence of pulmonary emboli detected by repeat lung scanning. ${ }^{10,11}$ Two meta-analysis do not support the systematic recommendation of bed rest as part of the early management of patients presenting with DVT, PE of both. $^{12,13}$

At present, research on bed rest in DVT patients focus on physiological outcomes with little attention on the psychological impact and quality of life implications of DVT. In the course of clinical practice, we found that these patients were often unable to immediately adapt to bed rest because of their level of consciousness and normal degree of physical activity. This seriously affected the QOL of these patients. Additionally, bed rest may even have negative consequences, such as work absenteeism and morbidity associated with immobilization. Hence, more substantive information is needed regarding bed rest as a treatment for acute DVT. This qualitative study explored the implications of bed rest from the perspective of patients with acute DVT. Understanding these implications will provide more evidence on whether bed rest should be used as a medical treatment of acute DVT.

\section{Patients and Methods}

\section{Study Design}

The phenomenological approach was used in order to understand the meaning and essence of participants' experiences in a given situation. In this approach, the given situation refers to the subjective feelings, perceptions, and reactions of the participants. ${ }^{14,15}$ For data collection, a descriptive qualitative design utilizing semistructured, in-depth, face-to-face interviews were conducted in order to explore the experience of absolute bed rest from the perspective of the patients with acute DVT. This design was chosen because it increased the understanding of the subjective experiences of the patients. In the future, this could be helpful in the development of qualitative tools and more categories of inquiry for researchers.

\section{Participants}

Hospitalized patients with acute DVT on absolute bed rest were recruited for this study through purposive sampling. As described by $\mathrm{Wu}$, purposive sampling, as a rule, is often used in qualitative research. ${ }^{16}$ Pursuant to the purpose of this study, only participants who could respond accurately to the research questions were selected. The inclusion criteria for participating in the study required that the patient should be (1) 18 years old or above, (2) on absolute bed rest for at least 14 days in order to treat acute DVT, (3) free of any history of VTE, tumor, surgery, or recent pregnancy, long-term bed rest, or childbirth, and (4) without any cognitive dysfunction or language disorder. These inclusion criteria will not only help researchers in understanding patient experiences but also present an insider's perspective of the experiences. The participants were chosen to be a representative sample of gender, age, education, and type of medical insurance.

\section{Interview Procedure and Data Analysis}

A preliminary draft of the interview regarding the bed rest experiences of patients with acute DVT was developed, as this has not been adopted in previous studies. Health professionals experienced in DVT patient care were consulted during this development. After pre-interviewing three participants, the draft was revised and the final interview schedule was created (Table 1).

Each interview was conducted by two researchers well versed in interview techniques. Participants were given a formal invitation and explanatory statement describing 
Table I Interview Schedule

Interview Schedule

I. What should you do according to your doctor's order of bed rest?

2. Can you talk about your bed rest experience?

3. How has bed rest changed or affected you and your life?

4. Do you have particular worries or difficulties as a result of bed rest?

5. What support do you expect during bed rest?

the interview procedures, risks and benefits, confidentiality, and anonymity of data.

Participants were recruited from the department of vascular surgery in the China-Japan Union Hospital of Jilin University between November and December, 2019. The interviews were conducted a day before the patients were discharged from hospital after the required 14 day stay in the hospital due to DVT. Each interview was generally conducted in a quiet room, lasted approximately 30 minutes, and was audio-recorded. Interviewers were instructed to react neutrally to the participants' answers and to remain sensitive and unbiased. Data collection was terminated when recurrent patterns became evident in participants' narrations. ${ }^{16}$ The audiotapes were transcribed into text within 48 hours of the interview by the researcher and another member of the research group. To ensure the authenticity of the data, transcriptions were then verified by the participants. Moreover, to identify the themes and subthemes emerging from the interviews for data analysis, the following seven steps in Colaizzi's descriptive phenomenological method were used to analyze the data and to find the themes and subthemes of the interviews: (1) familiarization, (2) identifying significant statements, (3) formulating meanings, (4) clustering themes, (5) developing an exhaustive description, (6) producing the fundamental structure, and (7) seeking verification of the fundamental structure. $^{17,18}$

As the first researcher of this study had long been exposed to the literature on bed rest, two other researchers conducted collaborative analysis in order to avoid unconsciously limiting views. This was done to expand the scope of the researchers' understanding and interpretation of the phenomenon. Three researchers performed independent data coding. In order to enhance credibility, theme development was achieved and consensus reached through at least two of these researchers collaborating during the three rounds of coding. In addition to this, two participants were asked to give their opinions on the analysis results.

\section{Ethics}

This study focused on clinical realities and was consistent with the Consolidated Criteria for Reporting Qualitative Research checklist ${ }^{19}$. Prior to the interviews, all participants provided verbal informed consent which was acceptable and approved by the Ethics Committees of China-Japan Union Hospital of Jilin University. Meanwhile, the patients informed consent included publication of anonymised quotes. Furthermore, this study was approved by the Ethics Committees of China-Japan Union Hospital of Jilin University (No. 2019082111).

\section{Results}

\section{Participant Characteristics}

Nine patients with acute DVT who had been put on bed rest were recruited. All the participants were treated with urokinase and heparin. The length of stay in the hospital ranged from 14 to 21 days. Detailed participant characteristics are described in Table 2.

\section{Emerging Themes}

Bed rest impaired the QOL of patients with acute DVT mainly across the physical, psychosocial, and social domains. Qualitative analysis revealed 12 subthemes and four main themes. The overall theme was "Implications of bed rest for patients with acute DVT." This overall theme was closely related to and influenced by the other four themes in complex ways (Figure 1).

\section{Theme I: Physical Effects}

\section{Subtheme I: Comfort Dropping}

Based on reports from the participants, symptoms experienced with acute DVT varied from severe to mild. Almost all participants described leg problems, specifically pain, swelling, and skin redness. Some participants reported that they frequently felt fatigue, limb numbness, and stiffness due to strict, long-term restrictions. Overall, the most common factor of these symptoms was that they were agonizing and disturbing, which negatively affected the patients' lives.

I had swelling and pain in my left leg. The doctor said there was a blood clot in it, which made it even more obvious that I felt something stuck in it. I also felt uncomfortable all the time. (Participant A)

\section{Subtheme 2: Impaired Sleep}

Bed rest greatly affected the patients' functionality. Sleep disturbances were common among the participants. They 
Table 2 Participant Characteristics

\begin{tabular}{|l|l|l|l|l|l|l|l|}
\hline ID & Age & Gender & Education & Occupation & Site of DVT & Localisation & Insurance Type \\
\hline A & 54 & Female & Primary school & Salesclerk & Left & Distal & City \\
B & 67 & Female & Technical secondary school & Retired & Left & Proximal & City \\
C & 66 & Female & Junior high school & Peasant & Both & Proximal & Rural Cooperative \\
D & $5 I$ & Male & Primary school & Driver & Right & Proximal & Province \\
E & 59 & Male & Junior high school & Worker & Left & Proximal & Self-paying \\
F & 64 & Male & Junior high school & Retired & Left & Distal & City \\
G & $4 I$ & Female & Primary school & Accountant & Both & All segments & City \\
H & 30 & Male & Illiterate & Office clerk & Right & Proximal & Province \\
I & 79 & Male & Senior high school & Retired & Left & Distal & City \\
\hline
\end{tabular}

Abbreviation: DVT, deep vein thrombosis.

also commonly reported factors that affected their sleep such as DVT symptoms, unfamiliar environment, and strict limitation of activity.

My first night in the hospital was quite difficult. I did not dare sleep as to prevent the movement of my leg. I did not sleep all night. (Participant C)

\section{Subtheme 3: Functional Limitations}

Generally, the participants reported that defecation on the bed was the most difficult aspect of bed rest. According to the doctor's instructions, patients on bed rest could not get up to use the bathroom. Therefore, they needed to urinate and defecate on the bed. Some patients reported that they had needed to urinate or defecate, but they could not due to the awkward defecation position, unfamiliar surroundings, and shyness.

I have no experience of defecating on the bed. There were so many people in the ward. Even though there are curtains, I still felt embarrassed and could not urinate. Because a long period passed without being able to urinate, the nurse needed to insert a urine tube in my body. (Participant G)

\section{Theme 2: Psychological Effects}

\section{Subtheme 4: Fear of Uncertainty of Disease} Progression and Fatal PE

Traditionally, patients with acute DVT had been taught that bed rest was to prevent dislodged clots from resulting in serious or fatal PE, which had a major impact on patients' lives and was perceived as life-threatening. It was common for most patients to have an intense fear of this serious consequence.

The doctor told me not to move, as the clot could fall. If so, I could die (pulmonary embolism and other serious complications). What I found on the Internet was consistent with what my doctor said. My daughter and I are scared. She even cried, although she did not do it in front of me. (Participant F)

\section{Subtheme 5: Anxiety}

Participants reported that significant levels of anxiety relating to bed rest were mostly due to the complications involved, such as pressure sores. They worried about the symptoms (post-thrombotic syndrome [PTS]) and possible lifelong treatment. They were also anxious about the recurrence of symptoms in the future, such as deterioration in QOL.

I begin to feel very uncomfortable about lying in bed without movement, as soon as I worry about sequelae. One night, I wanted to defecate and had been tolerant. However, I was afraid that the force of defecating would have an impact on my leg, which would lead to the moving of the blood clot. (Participant I)

\section{Subtheme 6: Impaired Self-Esteem}

Bed-rest-deprived patients with acute DVT of self-care ability and independence, which is not a good experience for these patients. They had to be highly conscious due to their being compelled to become dependent on others. They also felt a complete lack of privacy, as they were under watch by roommates and the nursing staff. These issues wounded their self-esteem and made patients feel frustrated.

I feel that I am a paralyzed patient during bed rest. There is nothing I can do. I could neither walk nor work. Eating, drinking, and defecating can only be carried out on the bed with the help of others. (Participant E)

\section{Subtheme 7: Self-Accusation and Guilt}

Participants, regardless of age, expressed self-accusation and guilt about being a burden to their loved ones while being on bed rest and, therefore, unable to perform normal 


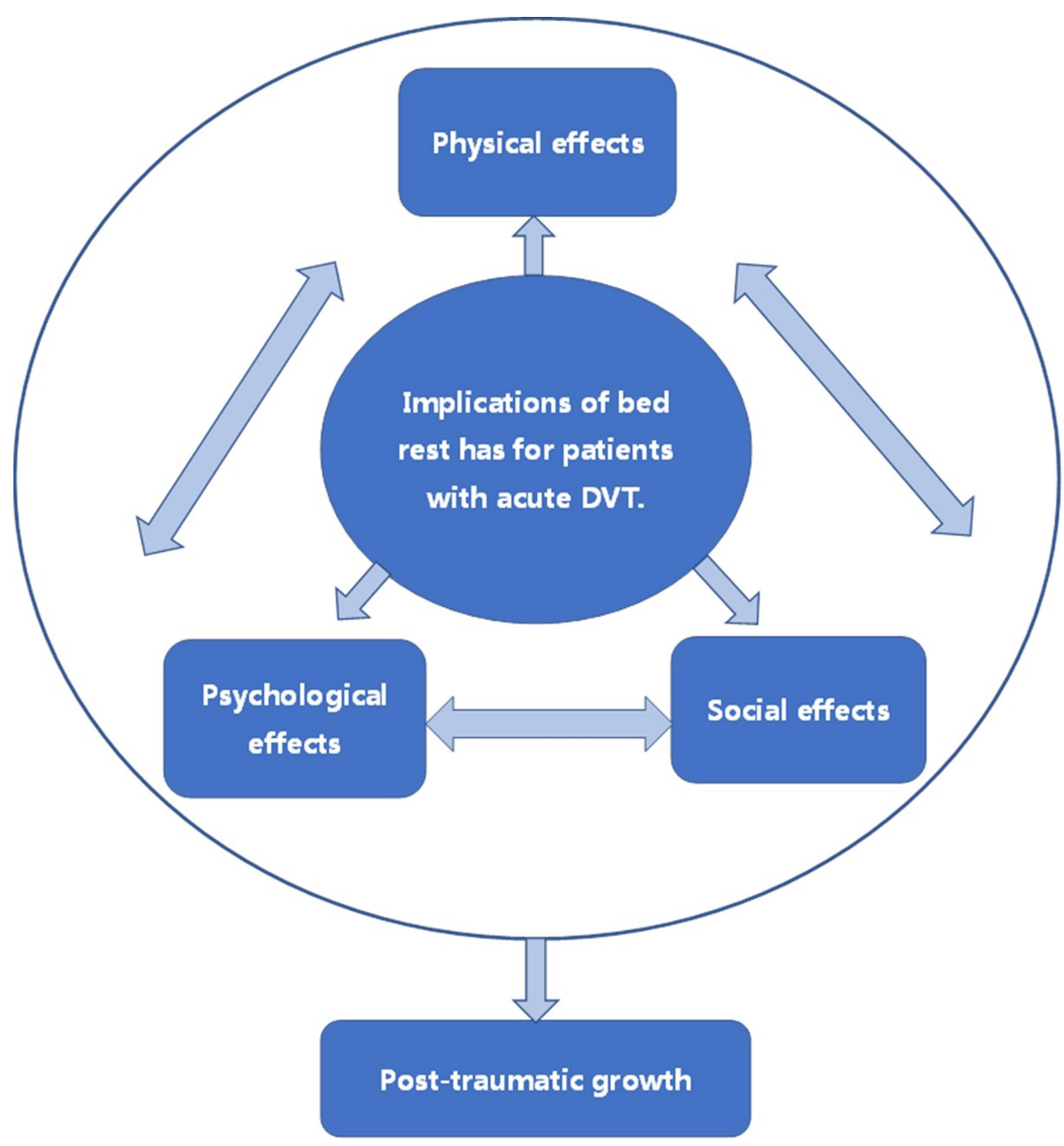

Figure I The main themes and their relationships to each other.

Abbreviation: DVT, deep vein thrombosis.

roles. Some participants felt guilty about their children, as their care needs subverted the normal order of parenting. It was now the children caring for the parent or the parent being unable to care for the children.

Because of DVT, I needed to take time off from work. The doctor asked me to stay in bed. Therefore, I needed someone to accompany me every day. My wife also took a leave from work to care for me. Our son was sent to his grandpa's home. I feel very guilty about being the one who causes all of the worry, extra work, exhaustion, and boredom. (Participant H)

\section{Theme 3: Social Effects Subtheme 8: Financial Distress}

Many participants discussed the financial distress associated with bed rest. They experienced reduced ability to work and generate income due to long hospitalization and immobilization after discharge. In addition, health insurance failed to fund or partially fund the expenses related to the cost of treatment. In most cases, self-employed patients lacked health insurance.

Because of DVT, I needed to stay in the hospital for a long time. Additionally, I needed to continue bed rest upon returning home after discharge. As such, I am unable to work for a long time and consequently have no income. This creates great financial pressure on me. (Participant D)

\section{Subtheme 9: Knowledge Deficit}

Most patients with acute DVT generally believed that medication, as opposed to bed rest, was the main treatment for DVT. Some patients had low compliance with bed rest, as they believed that doctors and nurses were exaggerating their illness and that occasional activity would not cause 
serious consequences. This indicated that patients lacked knowledge regarding DVT and absolute bed rest.

Is DVT serious? When I was in the hospital on the first day, the doctor criticized me because I was walking around the room instead of being on bed rest. I think the doctor made a mountain out of a molehill. (Participant C)

\section{Subtheme 10: Increased Reliance on Support from Others}

All participants reported that DVT affected their dependence. They relied on support from family, friends, and the medical and nursing staff. Some participants felt their health care providers did not grasp the effect bed rest had on their lives and families. Overall, the patients seemed to appreciate talking with the nursing staff and the researchers who could provide more knowledge on the disease.

Since I was diagnosed with DVT, I felt insecure all the time. I eagerly hoped that nurses could visit me and tell me more about the therapeutic method in conversation. I even wish that the doctors and nurses could pay a visit to me once an hour every day to check if my condition was getting better. (Participant B)

\section{Theme 4: Post-Trauma Growth}

\section{Subtheme II: Reflections on Life}

Participants began to re-examine life and display altruistic behavior due to their bed rest experience during acute DVT. The disease aroused a lot of feelings in the participants that allowed them to have a deeper understanding of life.

I now feel that life is fragile, so I must cherish it more. I used to live frugally, but I think I have to enjoy life from now on. (Participant A)

\section{Subtheme 12: Hope in Life}

After treatment, the symptoms of DVT gradually improved. This enabled participants to grow in confidence. They also longed to return to their normal state and to regain momentum in life.

After my recovery, I will no longer play mahjong every day. I want to have some exercise in order not to have a bed rest. (Participant E)

\section{Discussion}

Bed rest is recommended because of the concern of clot dislodgment by ambulation, causing a potentially fatal PE. ${ }^{12}$ This theoretical assumption is so simple that it might be viewed as "evidence-based." However, a metaanalysis, which included 13 studies with a total of 3269 patients, concluded that compared to bed rest, early ambulation of acute DVT patients with anticoagulation was not associated with a higher incidence of new PE, progression of DVT, and DVT related deaths. Furthermore, for the patients who experienced moderate or severe pain initially, a better outcome was seen in the early ambulation group regarding the remission of acute pain in the affected limb. ${ }^{13}$ Two earlier meta-analyses also did not support the recommendation of bed rest as part of the early management of patients presenting with DVT. ${ }^{12,20}$ However, the primary outcome measures of these studies were the incidence of new PE, progression of DVT, and DVT related deaths. No meta-analysis or systematic review of bed rest for acute DVT was available based on QOL. This qualitative study adds to the available evidence regarding the harm of bed rest as a treatment from the perspective of patients with acute DVT. These results indicate that bed rest which seriously affects the QOL of patients with acute DVT is not recommended and that it should no longer be part of the management or study of thromboembolic disorders.

Previous studies ${ }^{21,22}$ have reported the impact of DVT on QOL across different domains following the event. Most of those studies have examined the longitudinal change in QOL in DVT patients, or compared the effects of different DVT treatments. However, none of the studies have explored the experience of bed rest from the perspective of patients with acute DVT. The overarching themes from this qualitative study illustrate patients' experiences with bed rest and how bed rest affects their QOL. These themes were closely related to and influenced by each other in complex ways.

Absolute bed rest prevented patients with acute DVT from returning to normal life and daily activities independently. Due to long periods of bed rest, the patients suffered a decrease in comfort accompanied by sleep disorders and defecation disorders. Patients' normal needs conflict with the suddenly altered environment and they are unable to keep up with the pace of environmental changes, resulting in adverse consequences in terms of psychological and social adaptation. ${ }^{23}$ 
Bed rest has a considerable emotional effect on the patients. Patients on bed rest during acute DVT have negative mood alterations, such as fear, anxiety, impaired self-esteem, and self-accusation. Stressors come from both physical and social domains simultaneously, and physical and psychological effects continually interact. The lack of information and support about DVT and bed rest remained a source of negative emotions for patients. The suddenness and life-threatening nature of DVT formed a heavy load by contributing to episodes involving a vicious cycle of "post-thrombotic panic". ${ }^{24}$ Participants with little knowledge about DVT and bed rest hoped to seek information and medical help on the expected pathway to recovery immediately. Therefore, education and support are important with regard to coping with psychological pressure. Health professionals should tailor communication or adopt strategies to address specific needs for knowledge and support requirements of the patients consistently. ${ }^{25,26}$

Patients' family members or friends provided the greatest amount of assistance and support including direct care, comfort, and encouragement during bed rest, which may also reduce anxiety. The patients gained strength from people they relied on and their perceived support, which helped them cope with this disease. On the other hand, they were also concerned about burdening family and friends. Previous research supports the positive influence of family involvement during bed rest and showed that education of family members enhanced patient selfefficacy and reduced psychological pressure. ${ }^{27}$ Medical and nursing staff should encourage patients to involve significant others in their care because there are benefits to this increased involvement for acute DVT patients. Another study found that exposure to peers with similar experiences provided motivation and encouraged persistence. ${ }^{28}$ Connecting patients by means of WeChat groups, social networks, or support organizations may provide an additional source of support during bed rest for acute DVT patients. ${ }^{29}$

A surprising theme throughout this study was posttraumatic growth (PTG) by the participants, who take a fresh look at life and are hopeful for the future. Recovery presented enduring physical and psychological challenges, and coping with or overcoming them enabled participants to gain confidence and contributed to their search for meaning. However, the unique positive benefits to patients with DVT have been neglected in previous studies. Healthcare providers should appropriately guide the patients toward positive psychology and provide positive reinforcement under the premise of preventing negative emotions. ${ }^{30,31}$ The scale of post-traumatic growth could be also used to guide the patients toward producing a positive psychological experience.

This is the first qualitative study that explored patients' experience of bed rest in the acute DVT from the patients' perspective in the northeast of China. It adds to the available evidence for the harm of bed rest as a treatment from the perspective of patients with acute DVT and no similar study has also been conducted. By face-to-face and audiorecorded semi-structured interviews, we allowed patients with acute DVT to speak in their own voices about their experiences with bed rest, and we were able to delve deeper experiences and unmet clinical needs that emerged from interviews with participants, such as impaired selfesteem and self-accusation.

In considering the limitations of this study, it was a qualitative study exploring patients' experience of bed rest in the acute DVT at a single institution which limits interpretation and generalisability of the findings. However, other hospital settings can utilize our methods to learn similar lessons. A further limitation is we exclude the patients who had history of VTE, tumor, surgery, longterm bed rest, recent pregnancy and childbirth. This is because these patients may confuse the effects of other illnesses on the experience of bed rest. In the future, the experience of bed rest patients with comorbidities can be discussed on the basis of our findings and the individual situation.

Bed rest is so simple that it might be viewed as "evidence-based". However, it has not been proven scientifically. ${ }^{12}$ Researchers can continue to contribute to the body of knowledge on bed rest through further quantitative studies that focus on understanding the QOL on bed rest of the patients with acute DVT and reporting the difficulties related to this form of treatment. This information can then be analyzed in collaboration with medical colleagues to implement universal best practices.

\section{Conclusion}

Bed rest for patients with acute DVT is a physically, emotionally, and socially distressing phenomenon that simultaneously affects QOL and induces post-traumatic growth. We believe that bed rest is not beneficial to the physical and mental health of patients with acute DVT. This study adds to the available evidence on the harmful effect of bed rest as a medical treatment from the perspective of patients with acute DVT. Further quantitative studies should be conducted 
to compare the QOL and psychosocial status of patients with and without bed rest amidst acute DVT.

\section{Acknowledgments}

The authors would like to thank the nurses of Department of Vascular Surgery, China-Japan Union Hospital of Jilin University for providing a free room for interview and to thank all of the patients that took part in the study for sharing their experiences of bed rest.

\section{Funding}

This work was supported by the Graduate Innovation Fund of Jilin University [grant numbers 101832018C086]; the Education Department of Jilin Province [grant numbers JJKH20201125KJ]. The sponsor had no role in the study design, in the collection, analysis, and interpretation of the data, in the writing of this report, or in the decision to submit the paper for publication.

\section{Disclosure}

The authors declare that they have no conflicts of interest for this work.

\section{References}

1. Pillai AR, Raval JS. Does early ambulation increase the risk of pulmonary embolism in deep vein thrombosis? A review of the literature. Home Healthc Nurse. 2014;32:336-342. doi:10.1097/ NHH.0000000000000087

2. Heit JA, Spencer FA, White RH. The epidemiology of venous thromboembolism. J Thromb Thrombolysis. 2016;41:3-14.

3. Sun C, Wang J, Chang YM. Advances in epidemiological characteristics and risk factors of deep vein thrombosis (DVT) in China. J Qiqihar Med Coll. 2015;36:2282-2283.

4. Bell WR, Simon TL. Current status of pulmonary thromboembolic disease: pathophysiology, diagnosis, prevention, and treatment. $\mathrm{Am}$ Heart J. 1982;103:239-262. doi:10.1016/0002-8703(82)90498-7

5. Partsch H. Bed rest versus ambulation in the initial treatment of patients with proximal deep vein thrombosis. Curr Opin Pulm Med. 2002;8:389-393. doi:10.1097/00063198-200209000-00008

6. Sprague AE. The evolution of bed rest as a clinical intervention. J Obstet Gynecol Neonatal Nurs. 2004;33:542-549. doi:10.1177/ 0884217504268523

7. Winkelman C. Bed rest in health and critical illness: a body systems approach. AACN Adv Crit Care. 2009;20:254-266.

8. Browse NL. The physiology and pathology of bed rest. Am J Med Sci. 1965;250:228. doi:10.1097/00000441-196508000-00028

9. Lipnicki DM, Gunga HC. Physical inactivity and cognitive functioning: results from bed rest studies. Eur $J$ Appl Physiol. 2009;105:27-35. doi:10.1007/s00421-008-0869-5

10. Aschwanden M, Labs KH, Engel H, et al. Acute deep vein thrombosis: early mobilization does not increase the frequency of pulmonary embolism. Thromb Haemost. 2001;85:42-46. doi:10.1055/s-00371612901

11. Schellong SM, Schwarz TH, Krapp J, et al. Bed rest in deep vein thrombosis and the incidence of scintigraphic pulmonary embolism. Thromb Haemost. 1999;82:127-129. doi:10.1055/s-0037-1615570
12. Aissaoui N, Martins E, Mouly S, et al. A meta-analysis of bed rest versus early ambulation in the management of pulmonary embolism, deep vein thrombosis, or both. Int $J$ Cardiol. 2009;137(1):37-41. doi: $10.1016 /$ j.ijcard.2008.06.020

13. Zhenlei L, Xixi T, Yuexin C, et al. Bed rest versus early ambulation with standard anticoagulation in the management of deep vein thrombosis: a meta-analysis. PLoS One. 2015;10(4):e0121388. doi: 10.1371 /journal.pone. 0121388

14. Smith JA. Beyond the divide between cognition and discourse: using interpretative phenomenological analysis in health psychology. Psychol Health. 1996;11:261-271. doi:10.1080/08870449608400256

15. Brocki JM, Wearden AJ. A critical evaluation of the use of interpretative phenomenological analysis (IPA) in health psychology. Psychol Health. 2006;21:87-108. doi:10.1080/14768320500230185

16. Wu LJ, Huang HM, Lee HH. A comparison of convenience sampling and purposive sampling. J Nurs. 2014;61:105-111.

17. Christine W. Information point: Colaizzi's framework for analysing qualitative data. J Clin Nurs. 1999;8:576.

18. Edward KL, Welch T. The extension of Colaizzi's method of phenomenological enquiry. Contemp Nurse. 2011;39:163-171.

19. Buus N, Perron A. The quality of quality criteria: replicating the development of the consolidated criteria for reporting qualitative research (COREQ). Int J Nurs Stud. 2020;102:103452. doi:10.1016/ j.ijnurstu.2019.103452

20. Trujillo-Santos AJ, Martos-Pérez F, Perea-Milla E. Bed rest or early mobilisation as treatment of deep vein thrombosis: a systematic review and meta-analysis. Med Clín. 2004;17:641-647. doi:10.1016/S0025-7753(04)74340-7

21. Kahn SR, Ducruet T, Lamping DL, et al. Prospective evaluation of health-related quality of life in patients with deep venous thrombosis. Arch Intern Med. 2005;165:1173-1178. doi:10.1001/archinte. 165.10.1173

22. Van Es J, Den Exter PL, Kaptein AA, et al. Quality of life after pulmonary embolism as assessed with SF-36 and PEmb-QoL. Thromb Res. 2013;132:500-505. doi:10.1016/j.thromres.2013.06.016

23. O'Connell ME. Conflict and the environment (review). Hum Rights Q. 2000;22:1099-1103. doi:10.1353/hrq.2000.0047

24. Hunter R, Lewis S, Noble S, et al. "Post-thrombotic panic syndrome": a thematic analysis of the experience of venous thromboembolism. Br J Health Psychol. 2017;22:8-25.

25. Golemi I, Salazar Adum JP, Quintero LD, et al. Gaps of care in patients with venous thrombotic embolism: a qualitative study. Int Angiol. 2019;38:284-290. doi:10.23736/S0392-9590.19.04130-0

26. Benelhaj NB, Hutchinson A, Maraveyas AM, et al. Cancer patients' experiences of living with venous thromboembolism: a systematic review and qualitative thematic synthesis. Palliat Med. 2018;32:1010-1020. doi:10.1177/0269216318757133

27. Keefe FJ, Blumenthal J, Baucom D, et al. Effects of spouse-assisted coping skills training and exercise training in patients with osteoarthritic knee pain: a randomized controlled study. Pain. 2004;110:539-549. doi:10.1016/j.pain.2004.03.022

28. Watson NJ, Martin SA, Keating JL. The impact of wrist fracture, surgical repair and immobilization on patients: a qualitative study. Clin Rehabil. 2018;32:841-851. doi:10.1177/0269215518754614

29. Rubarth LB, Schoening AM, Cosiman A, Sandhurst H. Women's experience of hospitalized bed rest during high-risk pregnancy. J Obstet Gynecol Neonatal Nurs. 2012;41:398-407. doi:10.1111/ j.1552-6909.2012.01349.x

30. Hefferon K, Grealy M, Mutrie N. Post-traumatic growth and life threatening physical illness: a systematic review of the qualitative literature. Br J Health Psychol. 2019;14:343-378. doi:10.1348/ 135910708 X332936

31. Kreutzer L, Yang AD, Sansone C, et al. Barriers to providing VTE chemoprophylaxis to hospitalized patients: a nursing-focused qualitative evaluation. J Hosp Med. 2009;14:668-672. doi:10.12788/ jhm. 3290 


\section{Publish your work in this journal}

Patient Preference and Adherence is an international, peer-reviewed, open access journal that focusing on the growing importance of patient preference and adherence throughout the therapeutic continuum. Patient satisfaction, acceptability, quality of life, compliance, persistence and their role in developing new therapeutic modalities and compounds to optimize clinical outcomes for existing disease states are major areas of interest for the journal. This journal has been accepted for indexing on PubMed Central. The manuscript management system is completely online and includes a very quick and fair peer-review system, which is all easy to use. Visit http:// www.dovepress.com/testimonials.php to read real quotes from published authors. 